\title{
Does Geijera parviflora Lindl. (Rutaceae) facilitate understorey species in semi-arid Australia?
}

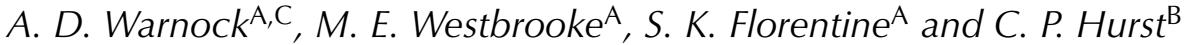 \\ ${ }^{A}$ Centre for Environmental Management, School of Science and Engineering, University of Ballarat, PO Box 663, \\ Victoria 3350, Australia. \\ ${ }^{B}$ Institute of Health and Biomedical Innovation, Queensland University of Technology, 60 Musk Avenue, \\ Kelvin Grove, Queensland 4059, Australia. \\ ${ }^{\mathrm{C} C}$ Corresponding author. Email: a.warnock@ballarat.edu.au
}

\section{Introduction}

Plant community composition under tree canopies often differs to that of surrounding micro-environments (Kennard and Walker 1973; Christie 1975; Haworth and McPherson 1994; Rousset and Lepart 2000; Choler et al. 2001; Hastwell 2001; Fensham and Butler 2003). While grass and herb biomass can be enhanced by the removal of trees in some areas through reduced competition, plant interactions is often positive in arid environments (Kennard and Walker 1973; Christie 1975; Florentine 1999; Rousset and Lepart 2000; Choler et al. 2001; Hastwell 2001; Fensham and Butler 2003; Armas and Pugnaire 2005). Plants in arid environments are often aggregated into islands surrounded by bare ground (Christie 1975; Ludwig et al. 2001; Bruno et al. 2003).

Overstorey species can directly facilitate understorey species through altering precipitation distribution under their canopy, soil bulk density, soil moisture, soil oxygen, soil and surface temperature, available light, soil and leaf litter accumulation, soil nutrient concentration and seed bank density (Belsky et al. 1993; Callaway 1994; Haworth and McPherson 1995; Florentine 1999; Ibáñez and Schupp 2001). Canopy species can also indirectly facilitate understorey plant growth and survival through protection from herbivores, increased microbial activity, overstorey-induced disturbance patterns or attracting pollinators (Callaway and Tyler 1996; Anderson et al. 2001; Smit et al. 2007). However, the effects of overstorey species on germination, survivorship and growth of understorey species can differ; some factors being facilitative, while others have little or negative effects (Rousset and Lepart 2000; Hastwell 2001).

Several field experiments have shown that positive effects of overstorey species increased as abiotic stress increases (Callaway 1997, 1998; Choler et al. 2001; Sthultz et al. 2007). In xeric environments, the effects of the overstorey were predominantly positive whereas in more mesic environments, competitive effects predominated (Berkowitz et al. 1995; Pugnaire and Haase 1996; Bruno et al. 2003; Sthultz et al. 2007). The 
balance between positive and negative effects on understorey species may also change seasonally. During periods of high temperatures and low rainfall, water is limited; therefore, shade from overstorey species reduce solar radiation, evaporation and evapotranspiration. During cooler periods with abundant moisture and cloud cover, light may become limited and shading may therefore have a negative effect (Hastwell and Facelli 2003).

Vegetation in arid zones is subject to short periods of abundant moisture. Trees can influence the distribution and longevity of soil moisture (Pressland 1973; Belsky et al. 1993; Belsky 1994; Caldwell et al. 1998; Anderson et al. 2001; Reinhart et al. 2006). Rainfall can be distributed towards the canopy edge via runoff or towards the tree bole via stemflow, creating spatial variation in soil moisture content under the canopy (Slatyer 1965; Haworth and McPherson 1995). Leaf litter under tree canopies also reduces evaporation facilitating growth and establishment of understorey species (Facelli and Pickett 1991).

Similar to many ecosystems, seed banks in arid ecosystems are characterised by high spatial variability. Low seed bank densities are associated with smooth bare soil surfaces such as those between canopies, whereas areas with perennial vegetation and leaf litter have much higher seed bank densities (Pugnaire and Lázaro 2000; Shaukat and Siddiqui 2004; Kinloch and Freidel 2005a). The effect of the canopy on soil seed banks adds to the facilitative effect of the microclimate created by the tree (Pugnaire and Lázaro 2000).

Soil physiochemical parameters and soil seed banks associated with Geijera parviflora Lindl. (wilga) in southwestern New South Wales, Australia, were investigated to determine whether they were associated with zonation of vegetation communities around the tree. The effects of artificial shading on species in a canopy-free area were investigated to understand how shading influences plant growth in isolation from other factors such as leaf litter accumulation and soil nutrient concentrations.

The study focused on the following questions: (i) does zonation of plant communities occur around $G$. parviflora; (ii) does zonation of soil physiochemical parameters occur around G. parviflora; (iii) does zonation of soil seed banks occur around G. parviflora; (iv) does G. parviflora redistribute precipitation, and (v) does artificial shading increase species abundance/biomass in a canopy-free environment.

\section{Methods}

Study site

The study of Geijera parviflora Lindl. and its associated understorey was located within a 2-ha vertebrate herbivore exclosure plot on Tarawi Nature Reserve, south-western New South Wales, Australia $\left(33^{\circ} 25^{\prime} 21^{\prime \prime} \mathrm{S}, 141^{\circ} 15^{\prime} 55^{\prime \prime} \mathrm{E}\right)$. The exclosure plot constructed in April 1991 was located within Casuarina pauper (belah) woodland with scattered G. parviflora. The G. parviflora specimens had a maximum height of $5 \mathrm{~m}$ and a maximum canopy radius of $4 \mathrm{~m}$ with the canopy foliage reaching within $2 \mathrm{~m}$ of the ground. Artificial shade plots were constructed in a cleared area of $C$. pauper woodland on Nanya Research Station, New South Wales ( $\left.33^{\circ} 12^{\prime} 08^{\prime \prime} \mathrm{S}, 141^{\circ} 17^{\prime} 04^{\prime \prime} \mathrm{E}\right), 20 \mathrm{~km}$ north of the exclusion plot. Botanical nomenclature is according to the Royal Botanic Gardens Sydney (2007).
The climate is classified as cool semi-arid (Dick 1975), being within the climatic zone 1B for New South Wales (Edwards 1979), with average daily maximum of $32^{\circ} \mathrm{C}$ in February and $15^{\circ} \mathrm{C}$ in July, and average daily minimum temperatures of $16^{\circ} \mathrm{C}$ in February and $5^{\circ} \mathrm{C}$ in July. Seasonal distribution of rainfall is fairly even but annual variation is high, the mean annual rainfall being $247 \mathrm{~mm}$ (T. Brown, pers. comm.).

\section{Sampling methods \\ Spatial heterogeneity of plant communities}

Five isolated $G$. parviflora trees were randomly selected within the herbivore exclosure plot. Species abundance and richness was recorded within $1 \mathrm{~m}^{2}$ quadrats from the following locations in the four cardinal directions: (i) beside the tree bole; (ii) mid-canopy, and (iii) $3 \mathrm{~m}$ from the canopy edge. Soil nutrient, soil moisture, soil seed bank samples and rain gauges were taken/positioned adjacent to each quadrat from the above positions. A total of 20 samples were taken for each variable.

\section{Spatial heterogeneity of soil nutrient concentrations}

Soil nutrient samples were taken $5-10 \mathrm{~cm}$ below the surface with a $10-\mathrm{cm}$ diameter soil corer. Sampling was conducted during autumn (April) and spring (September), 2005. Soil samples from each location were thoroughly mixed and a subsample sent for chemical analysis. Available $\mathrm{P}$ and $\mathrm{K}$ were measured using the Colwell (1965) method. Ammonium $\left(\mathrm{NH}_{4}{ }^{+}\right)$ and $\mathrm{NO}_{3}{ }^{-}$nitrogen were measured simultaneously using a Lachet Flow Injection analyser (Searle 1984). Combination electrodes were used to determine $\mathrm{pH}$ (both $\mathrm{H}_{2} \mathrm{O}$ and $\mathrm{CaCl}_{2}$ extract) and electrical conductivity calibrated against $0.01 \mathrm{M} \mathrm{KCl}$ (Rayment and Higginson 1992). Organic carbon was obtained by the Walkley and Black (1934) method and sulfur by the KCl-40 method (Blair et al. 1991). Iron was determined using a flame atomic absorption spectrophotometer at $248.3 \mathrm{~nm}$.

\section{Spatial heterogeneity of the germinable seed bank}

Sixty 1-kg soil seed bank samples including leaf litter were taken from a depth of $0-100 \mathrm{~mm}$ in April. Samples were mixed thoroughly, potted in $200 \times 150 \mathrm{~mm}$ seedling trays and placed in a glasshouse. Samples were watered for $1 \mathrm{~min}$ three times a day. All plants germinated were recorded weekly. After three months, species abundance in the germinable soil seed bank was calculated for each sample.

\section{Rainfall redistribution}

Rain gauges were positioned $500 \mathrm{~mm}$ above the soil surface. To prevent evaporation, $5 \mathrm{~mL}$ of vegetable oil was poured in the base of all gauges. Rain fell twice during the study, $3.6 \mathrm{~mm}$ in April and $70 \mathrm{~mm}$ in July. Precipitation redistributed to the soil adjacent to the tree bole via stemflow can often be measured by attaching a guttering system to the tree. But due to the coarse bark and scarring of $G$. parviflora trunks, it was not possible to use this method on trees at the study site and stemflow was therefore not measured.

\section{Spatial heterogeneity of soil moisture}

Soil moisture samples of $\sim 700 \mathrm{~g}$ were collected three weeks after rain in April and July. Samples were sealed in bags and 
taken back to the laboratory to be weighed, dried at $105^{\circ} \mathrm{C}$ for $24 \mathrm{~h}$ and reweighed. Percentage moisture was then calculated.

\section{Artificial shading trial}

Ten 90\%-, 50\%- and 0\%-light-exclusion shade plots were constructed in a canopy-free area on the 1 February 2005 at least $20 \mathrm{~m}$ from tree cover. Sclerolaena diacantha (grey copperburr), Dissocarpus paradoxus (cannonball burr) and Poa pratensis (Kentucky bluegrass) dominated this herbland before the plot construction. Shade mesh was attached to a $1.5-\mathrm{m}^{2}$ frame and positioned $0.5 \mathrm{~m}$ above the ground. Mesh fencing was used to exclude vertebrate herbivores from the plots. Species abundance, richness and Simpson's diversity (Simpson 1949) were recorded for all live vascular plant species within a $1-\mathrm{m}^{2}$ quadrat placed centrally in the exclosure plots after seven months of the shade treatment.

\section{Statistical analysis}

One-way ANOVAs were used to test for differences in soil nutrient concentrations, species richness, Simpson's diversity and soil moisture between location/shading treatments. ANOVAs were followed by Tukey's pair-wise comparison with Minitab 14.11 (Minitab Inc., State College, USA). Several soil nutrient variables failed to meet the assumption of equality of variance and normality, thus data were log-transformed and assumptions retested. All ANOVA assumptions were met before analysis.

The direct ordination method, Canonical Analysis of Principal Coordinates (CAP; Anderson and Willis 2003) was used to test for compositional differences among location/shading treatments and sampling period. This method was preferred as unlike any other direct ordination method, it allows flexibility in the selection of dissimilarity coefficient. In this particular analysis, the Bray-Curtis dissimilarity coefficient gave a superior performance and therefore was used in all subsequent multivariate analyses. All multivariate analysis was conducted using the Vegan R statistics package (version 2.1.1) and the R-library (Oksanen et al. 2006). Significance tests were conducted using permutation, and pair-wise comparisons were performed for each pair of treatments. Bonferroni (1936) adjustment was performed to ensure multiple comparisons did not lead to underestimated Type-I error rates. Taxa significantly correlated with the axis derived from CAP (those separating locations/treatments) were identified using the Envfit method from the Vegan package (Oksanen et al. 2006). Abundance data were log-transformed before CAP analysis to remove any large difference in scale among the original variables. All CAP assumptions were met before analysis.

\section{Results}

\section{Spatial heterogeneity of plant communities}

Plant community composition differed with position in relation to G. parviflora in both autumn $(F=5.13, P<0.05$; Fig. $1 a)$ and spring $(F=1.90, P<0.05$; Fig. $1 b)$. The zonation of plant communities was greater in spring, possibly a result of the preceding rainfall, season having a significant effect on the abundance of $47 \%$ of species $(F=9.22, P<0.05$, $n=18$ ). Differences were also found in species richness between
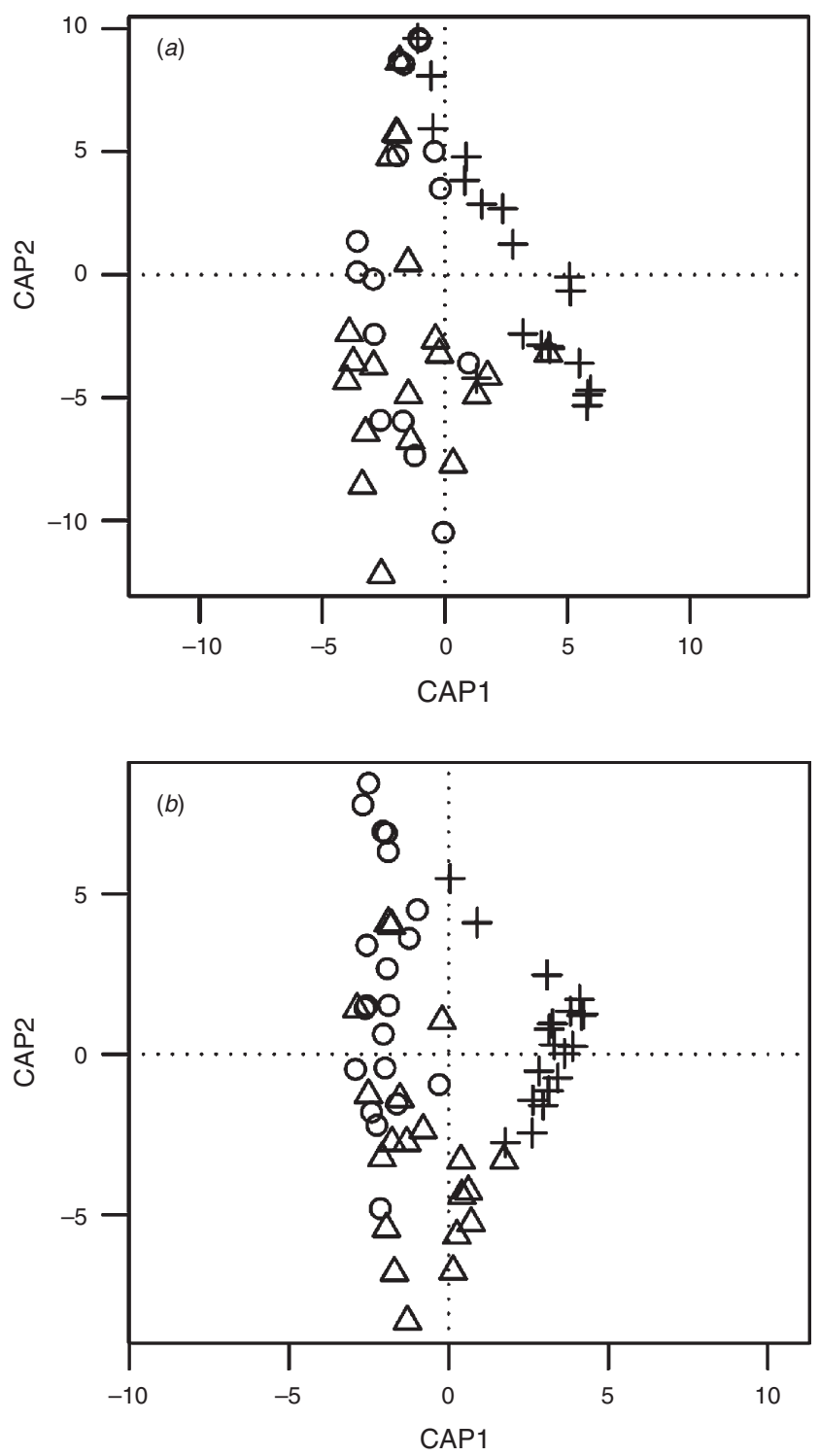

Fig. 1. Canonical Analysis of Principal Coordinates (CAP) ordination plots of species abundance in $(a)$ autumn and $(b)$ spring from $1 \mathrm{~m}^{2}$ quadrats taken from the Geijera parviflora tree bole $(O)$, mid-canopy $(\triangle)$, and $3 \mathrm{~m}$ from the canopy edge $(+)$.

locations in relation to $G$. parviflora during spring $(F=22.45$, $P<0.05$; Fig. $2 a$ ), but no difference was found during autumn $(F=2.42, P>0.05$; Fig. $2 b)$.

In autumn, both bole $(F=10.42, P<0.05 / 3)$ and midcanopy $(F=6.02, P<0.05 / 3)$ positions were different from the open position. But no difference in plant community composition was found between the two sampling positions under the canopy $(F=1.52, P>0.05)$. Dissocarpus paradoxus, Enchylaena tomentosa (ruby saltbush), Maireana pentatropis (erect mallee bluebush) and $S$. diacantha were found to be correlated with the CAP model $(P<0.05)$, i.e. position in relation to tree (Fig. 1a, Table 1). A high species score for D. paradoxus and low species scores for E. tomentosa, 


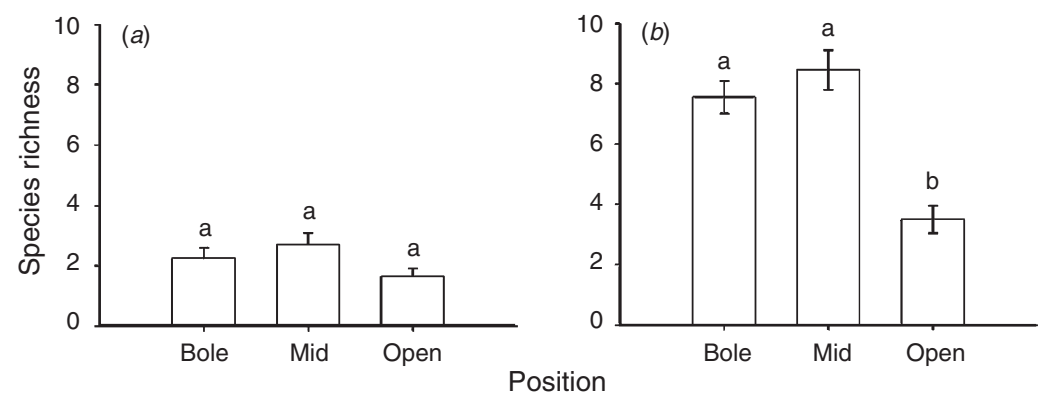

Fig. 2. Species richness at different positions in relationship to the canopy during (a) autumn and $(b)$ spring. Same letters not significantly different as determined by Tukey's pair-wise comparison. Error bars indicate \pm s.e.

Table 1. Mean densities \pm s.e. and correlation of species recorded from beside the Geijera parviflora tree bole, mid-canopy and $3 \mathrm{~m}$ from the canopy edge in autumn

$\mathrm{A} / \mathrm{P}=$ Annual/Perennial; position in relation to G. parviflora significant when $P<0.05$

\begin{tabular}{|c|c|c|c|c|c|c|c|c|}
\hline Family & Species & $\mathrm{A} / \mathrm{P}$ & Life form & $r^{2}$ & $P$ & Bole & Mid-canopy & Open \\
\hline Apocynaceae & Rhyncharrhena linearis & $\mathrm{P}$ & Climber & 0.010 & 0.815 & $0.00 \pm 0.00$ & $0.05 \pm 0.05$ & $0.05 \pm 0.05$ \\
\hline Asteraceae & Podolepis capillaris & $\mathrm{P}$ & Herb & 0.033 & 0.495 & $0.00 \pm 0.00$ & $0.00 \pm 0.00$ & $0.05 \pm 0.05$ \\
\hline Caesalpiniaceae & Senna artemisioides & $\mathrm{P}$ & Shrub & 0.011 & 0.856 & $0.00 \pm 0.00$ & $0.00 \pm 0.00$ & $0.10 \pm 0.10$ \\
\hline Caesalpiniaceae & Senna artemisioides subsp. petiolaris & $\mathrm{P}$ & Shrub & 0.071 & 0.126 & $0.55 \pm 0.31$ & $0.50 \pm 0.20$ & $0.05 \pm 0.05$ \\
\hline Chenopodiaceae & Atriplex semibaccata & $\mathrm{P}$ & Subshrub & 0.004 & 0.933 & $0.35 \pm 0.30$ & $0.35 \pm 0.35$ & $0.00 \pm 0.00$ \\
\hline Chenopodiaceae & Dissocarpus paradoxus & $\mathrm{P}$ & Subshrub & 0.243 & $<0.001$ & $0.10 \pm 0.10$ & $0.45 \pm 0.28$ & $2.20 \pm 1.08$ \\
\hline Chenopodiaceae & Einadia nutans & $\mathrm{P}$ & Subshrub & 0.031 & 0.513 & $0.00 \pm 0.00$ & $0.05 \pm 0.05$ & $0.00 \pm 0.00$ \\
\hline Chenopodiaceae & Enchylaena tomentosa & $\mathrm{P}$ & Subshrub & 0.409 & $<0.001$ & $1.75 \pm 0.28$ & $3.40 \pm 1.11$ & $0.85 \pm 0.28$ \\
\hline Chenopodiaceae & Maireana georgei & $\mathrm{P}$ & Subshrub & 0.018 & 0.745 & $0.00 \pm 0.00$ & $0.00 \pm 0.00$ & $0.15 \pm 0.15$ \\
\hline Chenopodiaceae & Maireana pentatropis & $\mathrm{P}$ & Subshrub & 0.639 & $<0.001$ & $12.3 \pm 6.83$ & $11.3 \pm 6.93$ & $0.00 \pm 0.00$ \\
\hline Chenopodiaceae & Sclerolaena diacantha & $P$ & Subshrub & 0.355 & $<0.001$ & $0.40 \pm 0.25$ & $1.60 \pm 0.85$ & $0.00 \pm 0.00$ \\
\hline Malvaceae & Sida corrugata & $P$ & Forb & 0.048 & 0.241 & $0.10 \pm 0.07$ & $0.00 \pm 0.00$ & $0.00 \pm 0.00$ \\
\hline Poaceae & Poa pratensis & $\mathrm{P}$ & Grass & 0.038 & 0.343 & $0.15 \pm 0.11$ & $0.10 \pm 0.07$ & $0.50 \pm 0.22$ \\
\hline Thymelaeaceae & Pimelea trichostachya & A & Herb & 0.029 & 0.45 & $0.05 \pm 0.05$ & $0.05 \pm 0.05$ & $0.05 \pm 0.00$ \\
\hline Zygophyllaceae & Zygophyllum ammophilum & A & Herb & 0.059 & 0.129 & $0.25 \pm 0.20$ & $0.00 \pm 0.00$ & $0.00 \pm 0.00$ \\
\hline
\end{tabular}

M. pentatropis and S. diacantha were found along the CAP1 axis of the ordination plot (by definition the axis showing greatest between group differences). This suggested that abundances of $E$. tomentosa, $M$. pentatropis and $S$. diacantha were higher under the canopy but $D$. paradoxus abundance appeared to be lower under the canopy.

In spring, both bole $(F=2.92, P<0.05 / 3)$ and mid-canopy ( $F=2.68, P<0.05 / 3)$ positions were different from the open position. But no difference in plant community composition was found between the two sampling positions under the canopy $(F=0.89, P>0.05)$. The species scores of the significantly correlated species associated with CAP1 of the ordination plot (Fig. $1 b$, Table 2), indicated that $38 \%(n=15)$ of species had higher abundances under the canopy. The plot also suggested that Crassula colorata, Chthonocephalus pseudevax (ground heads), $D$. paradoxus and $S$. diacantha have increased abundances beyond the canopy.

\section{Spatial heterogeneity of soil nutrient concentrations}

All soil-fertility indices except iron were higher under the canopy of $G$. parviflora $(P<0.05)$. No difference was found between the two under canopy positions $(P>0.05)$. No significant difference was found between mid-canopy and bole positions for any soil nutrients measured $(P>0.05)$. The results for soil nutrients are summarised in Table 3 .

\section{Spatial heterogeneity of the germinable seed bank}

The germinable seed bank composition differed with position in relation to the tree bole $(F=3.64, P<0.05)$, both bole $(F=7.12, P<0.05 / 3)$ and mid-canopy $(F=6.55, P<0.05 / 3)$ being different from the open position (Fig. 3). But no difference in seed bank composition was found between the two sampling positions under the canopy $(F=0.67, P>0.05)$. The species scores associated with the CAP1 axis (that separating canopy edge from sampling units closer to the bole) indicate that Calandrinia eremaea, Medicago minima (woolly burr medic), Oxalis perennans, Parietaria cardiostegia (mallee pellitory), P. pratensis, Tetragonia eremaea, Zygophyllum ammophilum (sand twinleaf) may have a higher germinable seed bank density under the canopy (Table 4).

\section{Rainfall redistribution}

G. parviflora was found to redistribute precipitation, reducing precipitation reaching the ground by $34.7 \%$ under the canopy 
Table 2. Mean densities \pm s.e. and correlation of species recorded from beside the Geijera parviflora tree bole, mid-canopy and $3 \mathrm{~m}$ from the canopy edge in spring

* = Introduced species; $\mathrm{A} / \mathrm{P}=\mathrm{Annual} / \mathrm{Perennial}$. Position in relation to G. parviflora significant when $P<0.05$

\begin{tabular}{|c|c|c|c|c|c|c|c|c|}
\hline Family & Species & $\mathrm{A} / \mathrm{P}$ & Life form & $r^{2}$ & $P$ & Bole & Mid-canopy & Open \\
\hline Aizoaceae & Tetragonia tetragonioides & $\mathrm{A}$ & Forb & 0.417 & $<0.001$ & $14.95 \pm 10.05$ & $19.60 \pm 10.08$ & $0.50 \pm 0.50$ \\
\hline Apocynaceae & Rhyncharrhena linearis & $\mathrm{P}$ & Climber & 0.193 & 0.001 & $1.00 \pm 0.57$ & $9.60 \pm 5.20$ & $0.00 \pm 0.00$ \\
\hline Asteraceae & Brachyscome lineariloba & $\mathrm{A}$ & Forb & 0.059 & 0.155 & $0.85 \pm 0.75$ & $0.45 \pm 0.40$ & $0.35 \pm 0.30$ \\
\hline Asteraceae & Chthonocephalus pseudevax & A & Forb & 0.187 & 0.003 & $0.00 \pm 0.00$ & $0.60 \pm 0.60$ & $20.05 \pm 14.93$ \\
\hline Asteraceae & Podolepis capillaris & $\mathrm{P}$ & Herb & 0.022 & 0.707 & $0.00 \pm 0.00$ & $0.05 \pm 0.05$ & $0.00 \pm 0.00$ \\
\hline Asteraceae & Vittadinia cuneata & $\mathrm{P}$ & Forb & 0.011 & 0.768 & $0.10 \pm 0.10$ & $0.05 \pm 0.05$ & $0.00 \pm 0.00$ \\
\hline Brassicaceae & Lepidium hyssopifolium & $\mathrm{P}$ & Forb & 0.261 & $<0.001$ & $12.00 \pm 5.13$ & $0.70 \pm 0.30$ & $0.05 \pm 0.05$ \\
\hline Asteraceae & Olearia pimeleoides & $\mathrm{P}$ & Shrub & 0.024 & 0.592 & $0.00 \pm 0.00$ & $0.00 \pm 0.00$ & $0.05 \pm 0.05$ \\
\hline Brassicaceae & Stenopetalum lineare & A & Forb & 0.055 & 0.174 & $0.30 \pm 0.25$ & $1.75 \pm 0.77$ & $0.00 \pm 0.00$ \\
\hline Chenopodiaceae & Atriplex semibaccata & $\mathrm{P}$ & Subshrub & 0.095 & 0.062 & $0.50 \pm 0.20$ & $0.00 \pm 0.00$ & $0.00 \pm 0.00$ \\
\hline Chenopodiaceae & Chenopodium curvispicatum & $\mathrm{P}$ & Subshrub & 0.149 & 0.008 & $0.55 \pm 0.17$ & $0.75 \pm 0.22$ & $0.10 \pm 0.07$ \\
\hline Chenopodiaceae & Chenopodium nitrariaceum & $P$ & Shrub & 0.026 & 0.527 & $0.05 \pm 0.05$ & $0.00 \pm 0.00$ & $0.00 \pm 0.00$ \\
\hline Chenopodiaceae & Dissocarpus paradoxus & $\mathrm{P}$ & Subshrub & 0.166 & 0.003 & $0.00 \pm 0.00$ & $0.30 \pm 0.22$ & $1.15 \pm 0.64$ \\
\hline Chenopodiaceae & Einadia nutans & $\mathrm{P}$ & Subshrub & 0.071 & 0.047 & $0.00 \pm 0.00$ & $0.05 \pm 0.05$ & $0.00 \pm 0.00$ \\
\hline Chenopodiaceae & Enchylaena tomentosa & $P$ & Subshrub & 0.216 & $<0.001$ & $1.05 \pm 0.25$ & $0.85 \pm 0.24$ & $0.05 \pm 0.05$ \\
\hline Chenopodiaceae & Maireana georgei & $\mathrm{P}$ & Subshrub & 0.057 & 0.206 & $0.10 \pm 0.07$ & $0.15 \pm 0.08$ & $0.15 \pm 0.08$ \\
\hline Chenopodiaceae & Maireana pentatropis & $\mathrm{P}$ & Subshrub & 0.514 & $<0.001$ & $5.80 \pm 2.36$ & $7.55 \pm 2.29$ & $0.00 \pm 0.00$ \\
\hline Chenopodiaceae & Maireana sedifolia & $P$ & Shrub & 0.071 & 0.047 & $0.00 \pm 0.00$ & $0.05 \pm 0.05$ & $0.00 \pm 0.00$ \\
\hline Chenopodiaceae & Sclerolaena diacantha & $\mathrm{P}$ & Subshrub & 0.135 & 0.012 & $0.50 \pm 0.35$ & $0.90 \pm 0.42$ & $0.70 \pm 0.42$ \\
\hline Crassulaceae & Crassula colorata & A & Forb & 0.738 & $<0.001$ & $7.50 \pm 7.50$ & $31.00 \pm 13.16$ & $122.00 \pm 31.57$ \\
\hline Fabaceae & Medicago minima* & A & Forb & 0.056 & 0.202 & $0.05 \pm 0.05$ & $0.10 \pm 0.07$ & $0.00 \pm 0.00$ \\
\hline Geraniaceae & Erodium crinitum & A & Forb & 0.057 & 0.164 & $0.00 \pm 0.00$ & $0.10 \pm 0.07$ & $0.00 \pm 0.00$ \\
\hline Malvaceae & Sida corrugata & $\mathrm{P}$ & Forb & 0.095 & 0.052 & $0.20 \pm 0.16$ & $0.45 \pm 0.19$ & $0.00 \pm 0.00$ \\
\hline Oxalidaceae & Oxalis perennans & $P$ & Forb & 0.076 & 0.095 & $0.10 \pm 0.07$ & $0.15 \pm 0.08$ & $0.00 \pm 0.00$ \\
\hline Poaceae & Poa pratensis & $\mathrm{P}$ & Grass & 0.455 & $<0.001$ & $8.25 \pm 2.57$ & $11.15 \pm 2.44$ & $2.15 \pm 0.70$ \\
\hline Portulaceae & Calandrinia eremaea & A & Forb & 0.116 & 0.032 & $28.15 \pm 12.72$ & $15.35 \pm 4.66$ & $6.95 \pm 2.44$ \\
\hline Portulaceae & Calotis hispidula & A & Forb & 0.205 & 0.002 & $0.80 \pm 0.75$ & $3.20 \pm 1.26$ & $0.10 \pm 0.07$ \\
\hline Rutaceae & Geijera parviflora & $P$ & Seedling & 0.077 & 0.1 & $0.15 \pm 0.08$ & $0.05 \pm 0.05$ & $0.00 \pm 0.00$ \\
\hline Sapindaceae & Dodonaea viscosa subsp. angustissima & $\mathrm{P}$ & Shrub & 0.033 & 0.454 & $0.00 \pm 0.00$ & $0.05 \pm 0.05$ & $0.05 \pm 0.05$ \\
\hline Thymelaeaceae & Pimelea microcephala subsp. microcephala & $\mathrm{P}$ & Shrub & 0.012 & 0.938 & $0.10 \pm 0.10$ & $0.00 \pm 0.00$ & $0.00 \pm 0.00$ \\
\hline Thymelaeaceae & Pimelea trichostachya & A & Herb & 0.047 & 0.241 & $0.05 \pm 0.05$ & $0.00 \pm 0.00$ & $0.00 \pm 0.00$ \\
\hline Urticaceae & Parietaria cardiostegia & A & Forb & 0.132 & 0.007 & $6.10 \pm 3.48$ & $1.00 \pm 0.59$ & $0.00 \pm 0.00$ \\
\hline Zygophyllaceae & Zygophyllum ammophilum & A & Herb & 0.466 & $<0.001$ & $19.85 \pm 6.46$ & $7.65 \pm 3.39$ & $0.00 \pm 0.00$ \\
\hline Zygophyllaceae & Zygophyllum eremaeum & A & Herb & 0.271 & $<0.001$ & $2.90 \pm 0.86$ & $3.55 \pm 1.60$ & $0.05 \pm 0.05$ \\
\hline
\end{tabular}

Table 3. Soil physio-chemical properties at three locations in relation to Geijera parviflora tree bole: beside the bole, mid-canopy and $3 \mathrm{~m}$ from canopy edge

Data are given as mean values \pm s.e. Means followed by the same letter are not significantly different $(P<0.05)$ as determined by Tukey's pair-wise comparison. $n=5$

\begin{tabular}{|c|c|c|c|c|c|}
\hline \multirow[t]{2}{*}{ Component } & \multicolumn{3}{|c|}{ Position } & \multirow[t]{2}{*}{$F$-value } & \multirow[t]{2}{*}{$P$} \\
\hline & Bole & Mid & Open & & \\
\hline $\mathrm{NH}_{4}{ }^{+}$nitrogen $(\mathrm{mg} / \mathrm{kg})$ & $72.4 \pm 3.62 \mathrm{a}$ & $46.4 \pm 4.23 \mathrm{a}$ & $2.4 \pm 0.06 \mathrm{~b}$ & 44.02 & $<0.001$ \\
\hline $\mathrm{NO}_{3}{ }^{-}$nitrogen $(\mathrm{mg} / \mathrm{kg})$ & $3.4 \pm 0.15 \mathrm{a}$ & $7.4 \pm 0.99 \mathrm{a}$ & $2.8 \pm 0.11 \mathrm{a}$ & 0.71 & 0.511 \\
\hline Phosphorus (mg/kg) & $42.6 \pm 1.00 \mathrm{a}$ & $29.4 \pm 1.51 \mathrm{a}$ & $10.4 \pm 0.15 b$ & 22.62 & $<0.001$ \\
\hline Potassium (mg/kg) & $590 \pm 11.49 a$ & $483.8 \pm 11.27 \mathrm{a}$ & $185.4 \pm 0.85 b$ & 68.65 & $<0.001$ \\
\hline Sulfur $(\mathrm{mg} / \mathrm{kg})$ & $17.4 \pm 0.93 \mathrm{a}$ & $13.84 \pm 0.59 \mathrm{a}$ & $1.04 \pm 0.01 b$ & 42.77 & $<0.001$ \\
\hline Organic carbon (\%) & $2.2 \pm 0.05 \mathrm{a}$ & $1.62 \pm 0.08 \mathrm{a}$ & $0.31 \pm 0.00$ & 30.4 & $<0.001$ \\
\hline Iron $(\mathrm{mg} / \mathrm{kg})$ & $427.8 \pm 3.29 \mathrm{a}$ & $472.8 \pm 3.02 \mathrm{ab}$ & $503.2 \pm 3.56 \mathrm{~b}$ & 6.57 & 0.012 \\
\hline Electrical conductivity $(\mathrm{dS} / \mathrm{m})$ & $0.34 \pm 0.01 \mathrm{a}$ & $0.21 \pm 0.01 \mathrm{a}$ & $0.02 \pm 0.00 \mathrm{~b}$ & 11.88 & $<0.001$ \\
\hline $\mathrm{pH}\left(\mathrm{CaCl}_{2}\right)$ & $7.4 \pm 0.02 \mathrm{a}$ & $7.26 \pm 0.03 a$ & $6.22 \pm 0.01 \mathrm{~b}$ & 57.61 & $<0.001$ \\
\hline $\mathrm{pH}\left(\mathrm{H}_{2} \mathrm{O}\right)$ & $8.04 \pm 0.02 \mathrm{a}$ & $7.94 \pm 0.04 a$ & $7.12 \pm 0.02 b$ & 16.73 & $<0.001$ \\
\hline
\end{tabular}

during the 3.6-mm rainfall event and $11.7 \%$ during the $70-\mathrm{mm}$ rainfall event. Canopy runoff was less than precipitation in open areas (Fig. 4).
Spatial heterogeneity of soil moisture

Higher soil moisture was found under the canopy of G. parviflora in April $(F=35.04, P<0.05)$ and July $(F=3.46, P<0.05)$, 


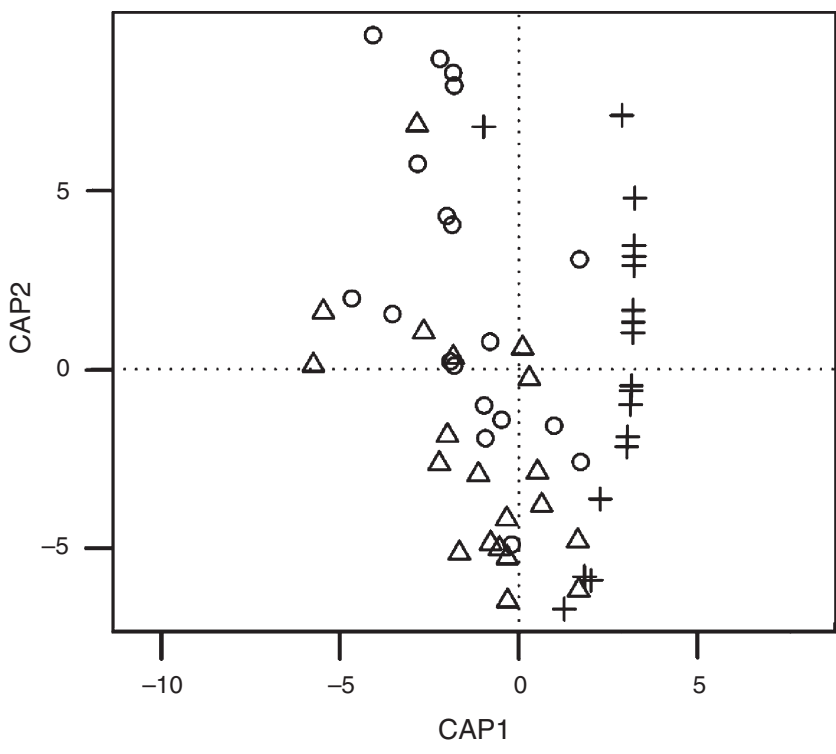

Fig. 3. Canonical Analysis of Principal Coordinates (CAP) ordination of species abundance from soil seed bank samples taken from beside the Geijera parviflora tree bole $(\mathrm{O})$, mid-canopy $(\triangle)$, and $3 \mathrm{~m}$ from the canopy $(+)$.

but distance from the bole within the canopy zone had no effect.

\section{Artificial shading trial}

Species richness differed significantly between shading treatments $(F=5.03, P<0.05)$. Shading increased species richness for both $50 \%(P<0.05 / 3)$ and $90 \%(P>0.05)$ shading treatments but no difference $(P>0.05 / 3)$ was found between the two treatments. Shading did not affect Simpson's index of diversity $(F=0.37, P>0.05 / 3)$.
The CAP analysis revealed a significant difference in composition associated with the shading effect $(F=1.23$, $P<0.05$; Fig. 5), but only between the control and $90 \%$ shading $(F=2.30, P<0.05 / 3)$.

All species with a significant correlation $(P<0.05)$ with the model had negative species scores on the CAP1 axis (Fig. 5), indicating that their respective abundances are positively associated with the degree of shading. These species being Brachyscome lineariloba (hard-headed daisy; $r^{2}=0.664$, $P<0.001)$, C. colorata $\left(r^{2}=0.290, P<0.05\right)$, Calotis hispidula (bogan flea; $\left.r^{2}=0.503, P<0.001\right)$, Daucus glochidiatus (native carrot; $\left.r^{2}=0.221, P<0.05\right), M$. minima $\left(r^{2}=0.664, P<0.001\right)$ and Scleranthus minusculus $\left(r^{2}=0.330, P<0.05\right)$.

\section{Discussion}

\section{Spatial heterogeneity of plant communities}

The study demonstrated that G. parviflora was associated with zonation of understorey vegetation. Two zones of understorey vegetation were found in relation to $G$. parviflora: (i) under the tree canopy with high species diversity, and (ii) beyond the canopy, this community being dominated by D. paradoxus throughout the year with $C$. colorata appearing after rainfall. Soil moisture, soil nutrient concentration and seed bank patterns also matched this zonation. This suggests that G. parviflora may create spatial heterogeneity of understorey plant communities over the broader environment.

Lower species richness found beyond tree canopies, may be associated with low soil nutrients coupled with high evaporation and evapotranspiration rates (Hastwell 2001). Many species are unable to cope with these conditions, whereas some such as $C$. pseudevax and D. paradoxus have adapted to moisture limited conditions. Common traits of xeric species such as reduced surface area of $C$. pseudevax and moisture-storing pubescent leaves of $D$. paradoxus aid in the reduction of water loss (Sandquist and Ehleringer 1998; Balsamo et al. 2003). Other

Table 4. Mean seed bank density $\left(\mathrm{kg}^{-2}\right)$ of soil samples taken from beside the Geijera parviflora tree bole, mid-canopy and $3 \mathrm{~m}$ from the canopy edge

Samples taken from $0-10 \mathrm{~cm}$ including leaf litter. ${ }^{*}=$ Introduced species; $\mathrm{A} / \mathrm{P}=$ Annual/Perennial. Position in relation to G. parviflora significant when $P<0.05$

\begin{tabular}{|c|c|c|c|c|c|c|c|c|}
\hline \multirow[t]{2}{*}{ Family } & \multirow[t]{2}{*}{ Species } & \multirow[t]{2}{*}{$\mathrm{A} / \mathrm{P}$} & \multirow[t]{2}{*}{ Life form } & \multirow[t]{2}{*}{$r^{2}$} & \multirow[t]{2}{*}{$P$} & \multicolumn{3}{|c|}{ Mean seed bank density $\left(\mathrm{kg}^{-2} \pm\right.$ s.e. $)$} \\
\hline & & & & & & Bole & Mid & Open \\
\hline Aizoaceae & Tetragonia eremaea & A & Forb & 0.1419 & 0.010 & $1.74 \pm 3.23$ & $1.55 \pm 3.25$ & $0.05 \pm 0.23$ \\
\hline Asteraceae & Angianthus sp. & A & Herb & 0.0123 & 0.740 & $0.00 \pm 0.00$ & $0.15 \pm 0.37$ & $0.00 \pm 0.00$ \\
\hline Asteraceae & Brachyscome lineariloba & A & Forb & 0.0079 & 0.831 & $0.26 \pm 0.65$ & $0.00 \pm 0.00$ & $0.00 \pm 0.00$ \\
\hline Brassicaceae & Lepidium hyssopifolium & $\mathrm{P}$ & Forb & 0.0674 & 0.129 & $0.74 \pm 1.52$ & $0.05 \pm 0.22$ & $0.00 \pm 0.00$ \\
\hline Casuarinaceae & Casuarina pauper & $\mathrm{P}$ & Tree & 0.0347 & 0.428 & $0.11 \pm 0.46$ & $0.00 \pm 0.00$ & $0.00 \pm 0.00$ \\
\hline Chenopodiaceae & Einadia nutans & $\mathrm{P}$ & Subshrub & 0.0888 & 0.068 & $0.26 \pm 0.45$ & $0.15 \pm 0.37$ & $0.00 \pm 0.00$ \\
\hline Chenopodiaceae & Maireana pentatropis & $\mathrm{P}$ & Subshrub & 0.0309 & 0.472 & $0.05 \pm 0.23$ & $0.20 \pm 0.70$ & $0.00 \pm 0.00$ \\
\hline Crassulaceae & Crassula colorata & A & Forb & 0.7397 & $<0.001$ & $13.95 \pm 17.93$ & $39.20 \pm 45.06$ & $23.26 \pm 21.08$ \\
\hline Fabaceae & Medicago minima* & A & Forb & 0.2391 & $<0.001$ & $0.16 \pm 0.37$ & $0.05 \pm 0.22$ & $0.00 \pm 0.00$ \\
\hline Oxalidaceae & Oxalis perennans & $\mathrm{P}$ & Forb & 0.1614 & 0.007 & $0.63 \pm 1.01$ & $0.45 \pm 0.89$ & $0.00 \pm 0.00$ \\
\hline Poaceae & Poa pratensis & $\mathrm{P}$ & Grass & 0.1485 & 0.011 & $2.42 \pm 2.32$ & $2.70 \pm 3.77$ & $0.00 \pm 0.00$ \\
\hline Portulaceae & Calandrinia eremaea & A & Forb & 0.1540 & 0.002 & $1.74 \pm 3.03$ & $2.30 \pm 2.36$ & $1.47 \pm 5.48$ \\
\hline Portulaceae & Calotis hispidula & A & Forb & 0.0440 & 0.307 & $0.05 \pm 0.23$ & $0.15 \pm 0.49$ & $0.00 \pm 0.00$ \\
\hline Urticaceae & Parietaria cardiostegia & A & Forb & 0.4251 & $<0.001$ & $0.89 \pm 1.56$ & $1.95 \pm 3.65$ & $0.00 \pm 0.00$ \\
\hline Zygophyllaceae & Zygophyllum ammophilum & A & Herb & 0.3798 & $<0.001$ & $2.26 \pm 3.11$ & $1.15 \pm 2.01$ & $0.00 \pm 0.00$ \\
\hline Zygophyllaceae & Zygophyllum eremaeum & A & Herb & 0.0037 & 0.896 & $0.21 \pm 0.54$ & $0.65 \pm 1.09$ & $0.00 \pm 0.00$ \\
\hline
\end{tabular}



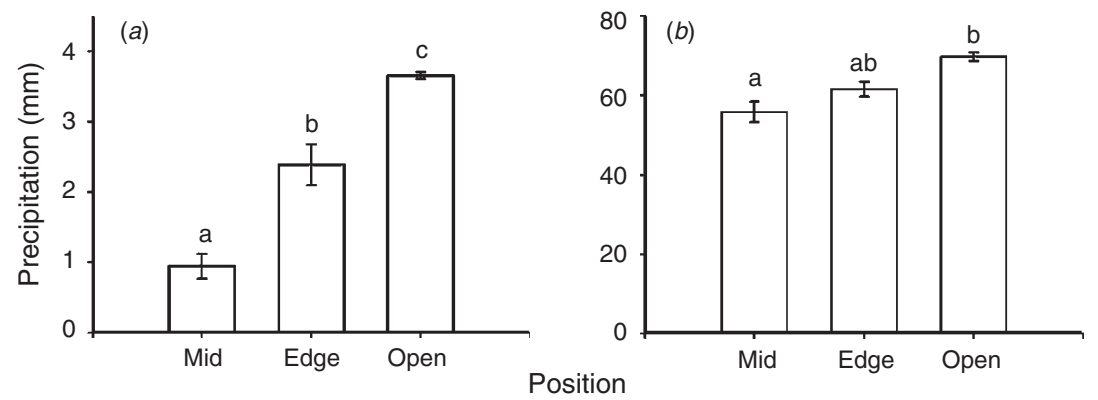

Fig. 4. Distribution of rainfall in different positions in relationship to the canopy during (a) a 3.6-mm rainfall event in April and (b) a 70-mm rainfall event in July. Same letters not significantly different as determined by Tukey's pair-wise comparison. Error bars indicate \pm s.e.

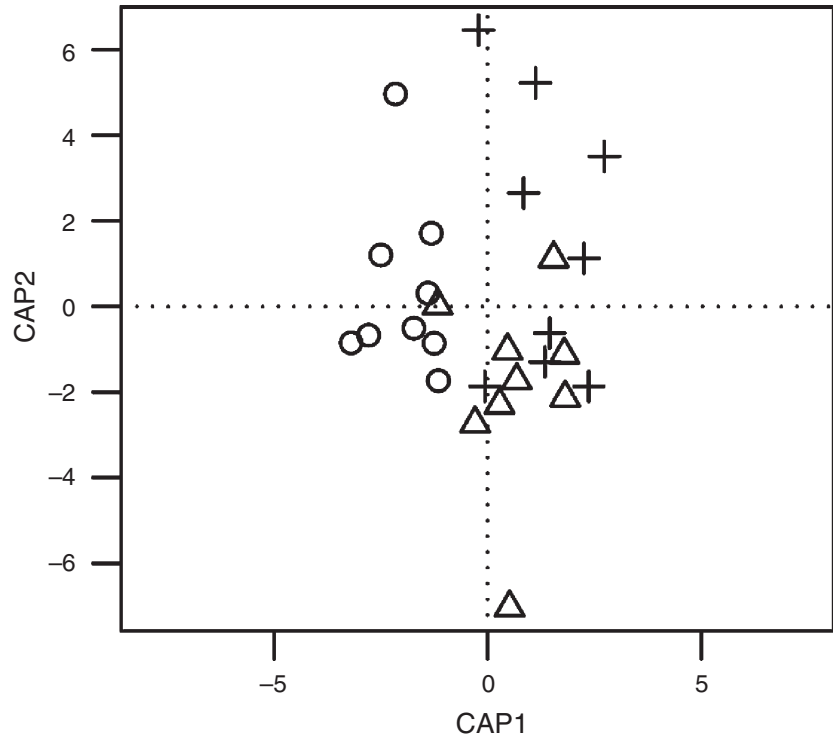

Fig. 5. Canonical Analysis of Principal Coordinates (CAP) ordination plot of species abundance from $0 \%(O), 50 \%(\triangle)$ and $90 \%(+)$ shade exclusion plots.

more mesophytic plants, such as $P$. cardiostegia, are only found in the microclimate created by the tree, being less adapted to moisture limited environments.

Increased abundance of some species under the tree may also be explained by an increased soil seed bank, seed dispersing fauna often spending much of the day in the shade of trees or perched in the canopy (Shaukat and Siddiqui 2004). Therefore, seeds are more likely to be dispersed in such areas, particularly species with fleshy fruits such as E. tomentosa. Leaf litter accumulation under the canopy may act as a trap for winddispersed seeds such as M. pentatropis, increasing soil seed bank densities under tree canopies.

High rainfall was recorded before the spring-sampling period. These seasonal differences were correlated with the composition of the understorey plant community beneath the canopy. But these seasonal differences plant composition were not reflected in the plant community beyond the canopy. The lack of seasonal differences in species composition beyond the canopy was possibly due to the low soil seed bank in this zone, resulting in few species germinating in this zone after rain. Seedlings that emerge beyond the canopy must also withstand high evaporation and evapotranspiration rates (Hastwell 2001; Heinemann and Kitzberger 2006) resulting in plants dying before maturity.

It was not possible to measure plant communities and soil physiochemical parameters beyond $3 \mathrm{~m}$ from the canopy edge, as samples beyond this distance would infringe on canopies and root zones of surrounding trees, therefore biasing data. The samples taken from beyond the tree canopy may still be within the trees' root zone, consequently being negatively effected due to depletion of nutrients and soil moisture exaggerating the positive effects of the tree (Arnold 1964; Tiedemann and Klemmedson 1973; Belsky et al. 1989; Vetaas 1992; Belsky 1994; Haworth and McPherson 1995; Song et al. 2000). The root zone of adjacent trees at the study site may overlap; this would leave no area unaffected by the tree canopy. It was not known whether the roots of G. parviflora affect understorey species beyond the canopy radius at the study site. Further study is necessary to discover the extent of the root zone of arid-zone trees such as $G$. parviflora and their zones of effect.

As the study was conducted within a 2-ha herbivore exclosure plot, the results from this study should be interpreted with caution when applied to a broader scale.

\section{Spatial heterogeneity of soil nutrient concentrations}

G. parviflora was found to be associated with spatial heterogeneity of soil nutrients. Except for iron, concentrations of all soil nutrients were higher under the canopy. This heterogeneity of soil nutrient concentration was likely to be a result of acquiring nutrients within the root zone both from beyond the canopy and beyond the root depth of many understorey species. The nutrients were then redistributed through litterfall (Callaway et al. 1991) and fine root turnover (Muñoz and Beer 2001), concentrating the nutrients under the canopy at a depth accessible to shrubs, herbs and grasses.

Indirect nutrient addition may take place due to faunal use of the tree canopy, leading to deposition of faecal matter and dead insects (Taylor and Hedges 1984). Leaf litter under the tree canopies may act as a trap collecting blown litter and increasing soil nutrient levels. Understorey species can take advantage of these nutrients, possibly explaining higher abundances of species under the canopy. Although lower soil nutrient concentrations were found in canopy-free areas, more study is needed to confirm 
whether low soil nutrient concentration limits plant growth beyond the canopy.

Previous studies have shown that increased water content of soils allowed a greater iron uptake of plants, particularly in areas with high organic matter content (Jones 1972, 1973). Soil moisture and organic carbon were higher under the canopy of G. parviflora which may explain the reduced iron content in soil under the canopy. The high abundance of understorey species and the overstorey itself may take up the soluble iron under the canopy, reducing its availability in the soil.

One limitation of this study was that it is not known whether $G$. parviflora created the spatial heterogeneity of nutrient concentrations or whether the tree simply germinated in more favourable patches. Without a long-term repeatedmeasure study of nutrient concentrations under immature trees, it would be impossible to confirm this. In this study, we assumed that $G$. parviflora created changes in nutrient concentrations associated with the tree canopy.

The spatial heterogeneity of soil nutrient concentration associated with $G$. parviflora may be a key factor influencing differences in plant communities in relation to its canopy.

\section{Spatial heterogeneity of the germinable seed bank}

The results indicated that the tree canopy of G. parviflora has an important influence on the germinable soil seed bank, possibly increasing the germinable seed bank of $C$. eremea, $M$. minima, $O$. perennans, $P$. cardiostegia, $P$. pratensis, T. eremaea and $Z$. eremaeum under the canopy.

Seeds are usually dispersed by animals, wind or water (Callaway 1994; Silvertown and Wilson 1994; Fensham and Butler 2003). Seeds enclosed in a sticky or prickly fruit show adaptations to animal dispersal. The sticky or prickly fruits of species such as $M$. minima and $P$. cardiostegia are caught in the fur of macropods or rodents and deposited under tree canopies while they rest in the shade/cover of the tree (Silvertown and Wilson 1994; Whitford 2002). Seeds enclosed in a fleshy fruit such as E. tomentosa are also dispersed through animal faeces. The seeds of these plants may be deposited by small mammals or birds as they perch in the above-canopy or rest in the cover of the tree (Shaukat and Siddiqui 2004). Herbivores can have had a strong influence in shaping vegetation communities (Silvertown and Wilson 1994; Rousset and Lepart 1999, 2000; Whitford 2002; Brooker et al. 2006). As the study was conducted within a 14-year-old herbivore exclosure plot, the community was no longer influenced by herbivore disturbance. However, the plant community may have been shaped by herbivores before plot construction. Seeds transported by herbivores before plot construction were able to germinate under the microclimate created by the canopy. As many seeds fall close to the mother plant, the effect of herbivore disturbance on the germinable seed bank may last several generations (Callaway 1994; Silvertown and Wilson 1994; Fensham and Butler 2003).

The winged fruit of species such as $Z$. eremaeum are adapted to wind dispersal. These seeds also often accumulate under tree canopies (Silvertown and Wilson 1994; Whitford 2002). As the seeds are blown along the bare ground, they are caught in the leaf litter under the canopies of trees (Shaukat and Siddiqui 2004).

E. tomentosa may not have been recorded in the seed bank at the time of study even though it was abundant under the canopy due to this high seasonal variability of transient soil seed banks (Auld 1995; Funes et al. 2001). Seed banks from most habitats contain both transient and persistent components (Auld 1995; Funes et al. 2001). The transient component consists of shortlived, non-dormant seeds which may not be viable at the onset of the second growing season. The persistent component consists of seeds from the current year as well as viable dormant seeds from previous years. Soil seed banks at certain times of the year may only contain persistent seeds as all transient seeds have either germinated or are no longer viable (Shaukat and Siddiqui 2004). The abundance of persistent and transient seeds in the soil seed bank can be strongly affected by seasonal conditions, thus seed bank assessments should be conducted over several years (Kinloch and Freidel 2005a, 2005b). As this study only examined the transient seed bank, caution should be taken when interpreting results.

\section{Rainfall redistribution}

G. parviflora canopies reduced direct precipitation in low rainfall events under the canopy due to interception by the canopy. When precipitation is intercepted by the canopy in low rainfall events, it often evaporates from the canopy leaves before reaching the ground (Slatyer 1965). This may negatively affect soil moisture under the canopy. But the effects of canopy interception are often masked during larger rainfall events due to the higher runoff (Pressland 1973, 1976).

Percent throughfall and canopy runoff are not always linearly related to rainfall intensity (Pressland 1973, 1976). For this reason, further study is needed to discover the effect of G. parviflora canopies on precipitation redistribution in various rainfall intensities as only two events occurred during the study period.

\section{Spatial heterogeneity of soil moisture}

Higher soil moisture was found at both the tree bole and midcanopy even though canopy interception reduced precipitation. This was likely to be a result of decreased evaporation due to shading, leaf litter cover, hydraulic lift (Song et al. 2000; Zou et al. 2005) or increased soil permeability under the canopy (Perrolf and Sandström 1995). However, hydraulic lift and soil permeability were not tested in this study.

\section{Artificial shading trial}

The increased abundance of $B$. lineariloba, C. colorata, C. hispidula, D. glochidiatus, M. minima and S. minusculus under the $90 \%$-shading treatment showed that shading was an important factor in the growth and survival of understorey species. The shade allowed more mesophytic species to colonise. The species that increased in abundance as a result of the shading treatment were not found in abundance under the canopy of $G$. parviflora. Nevertheless, the shading treatment gave an understanding of the importance of shade in facilitating understorey growth. The shading treatment was in isolation from other factors influencing understorey species such as canopy-related seed dispersal and depth of litter. It was also early in the successional process after the addition of shade as it was constructed seven months before sampling. A similar community to that under the canopy of $G$. parviflora may result given a longer successional period and the addition of other 
influential factors. It is not known, however, how long species will survive in the microclimate created by shade and they may die off in drier months. This seasonal variability was found under the tree canopies where in earlier drier months, few species were present despite the effect of shading.

\section{Conclusions}

G. parviflora was associated with zonation of understorey vegetation, suggesting that it generated spatial heterogeneity over the broader plant community and improved the establishment and survival of many understorey species thus increasing species diversity. These differences were particularly noticeable following rainfall events. Species with poor drought tolerance were able to establish after a precipitation event under the tree canopy which retained soil moisture, shade and increased leaf litter cover, reducing evaporation and evapotranspiration (Hastwell 2001; Hastwell and Facelli 2003). Positive effects of the tree on soil moisture such as shading outweighed negative effects such as canopy interception of rainfall and competition, particularly during large $(\sim 70 \mathrm{~mm})$ rainfall events. More study is needed on its effect on differing rainfall intensities. Shading appears to be a key influence in facilitating the establishment and growth of understorey species. This emphasises the importance of arid-zone trees in conserving understorey plant diversity.

\section{Acknowledgements}

The authors thank the University of Ballarat for financial support and the provision of laboratory facilities, and Jo Gorman and John Warren of New South Wales National Parks and Wildlife Service for access to sites within Tarawi Nature Reserve. We are also grateful to students from the University of Ballarat who assisted with field work. Kate Gosney provided additional help with statistical analysis. John Pickard and an anonymous reviewer provided useful comments on an earlier draft of the manuscript.

\section{References}

Anderson, L. J., Brumbaugh, M. S., and Jackson, R. B. (2001). Water and tree-understorey interactions: a natural experiment in a savanna with oak wilt. Ecology 82, 33-49.

Anderson, M. J., and Willis, T. J. (2003). Canonical analysis of principal coordinates: a new ecologically meaningful approach for constrained ordination. Ecology 84, 511-525. doi: 10.1890/00129658(2003)084[0511:CAOPCA]2.0.CO;2

Armas, C., and Pugnaire, F. I. (2005). Plant interactions govern population dynamics in a semi-arid plant community. Journal of Ecology 93, 978-989. doi: 10.1111/j.1365-2745.2005.01033.x

Arnold, J. F. (1964). Zonation of understory vegetation around a juniper tree. Journal of Range Management 47, 41-42.

Auld, T. D. (1995). Soil seedbank patterns of four trees and shrubs from arid Australia. Journal of Arid Environments 29, 33-45. doi: 10.1016/S01401963(95)80062-X

Balsamo, R. A., Bauer, A. M., Davis, S. D., and Rice, B. M. (2003). Leaf biomechanics, morphology, and anatomy of the deciduous mesophyte Prunus serrulata (Rosaceae) and the evergreen sclerophyllous shrub Heteromeles arbutifolia (Rosaceae). American Journal of Botany 90, 72-77.

Belsky, A. J. (1994). Influences of trees on savanna productivity: tests of shade, nutrients, and tree-grass competition. Ecology 75, 922-932. doi: $10.2307 / 1939416$

Belsky, A. J., Amundson, R. G., Duxbury, J. M., Riha, S. J., Ali, A. R., and Mwonga, S. M. (1989). The effects of trees on their physical, chemical, and biological environments in a semi-arid savanna in Kenya. Journal of Applied Ecology 26, 1005-1024. doi: 10.2307/2403708
Belsky, A. J., Mwonga, S. M., Amundson, R. G., Duxbury, J. M., and Ali, A. R. (1993). Comparative effects of isolated trees on their undercanopy environments in high and low-rainfall savannas. Journal of Applied Ecology 30, 143-155. doi: 10.2307/2404278

Berkowitz, A. R., Canham, C. D., and Kelly, V. R. (1995). Competition vs. facilitation of tree seedling growth and survival in early successional communities. Ecology 76, 1156-1168. doi: 10.2307/1940923

Blair, G. J., Chinoim, N., Lefroy, D. B., Anderson, G. C., and Crocker, G. J. (1991). A sulfur test for pastures and crops. Australian Journal of Soil Research 29, 619-626. doi: 10.1071/SR9910619

Bonferroni, C. E. (1936). Teoria statistica delle classi e calcolo delle probabilit à. Pubblicazioni del $R$ Istituto Superiore di Scienze Economiche e Commerciali di Firenze 8, 3-62.

Brooker, R. W., Scott, D., Palmer, S. C. F., and Swaine, E. (2006). Transient facilitative effects of heather on Scots pine along a grazing disturbance gradient in Scottish moorland. Journal of Ecology 94, 637-645. doi: 10.1111/j.1365-2745.2006.01129.x

Bruno, J. F., Stachowicz, J. J., and Bertness, M. D. (2003). Inclusion of facilitation into ecological theory. Trends in Ecology \& Evolution 18, 119-125. doi: 10.1016/S0169-5347(02)00045-9

Caldwell, M. M., Dawson, T. E., and Richards, J. H. (1998). Hydraulic lift: consequences of water efflux from the roots of plants. Oecologia 113, 151-161. doi: 10.1007/s004420050363

Callaway, R. M. (1994). Facilitative and interfering effects of Arthrocnemum subterminale on winter annuals. Ecology $\mathbf{7 5}$, 681-686. doi: 10.2307/1941726

Callaway, R. M. (1997). Positive interactions in plant communities and the individualistic-continuum concept. Oecologia 112, 143-149. doi: 10.1007/s004420050293

Callaway, R. M. (1998). Competition and facilitation on elevation gradients in subalpine forests of the northern Rocky Mountains, USA. Oikos 82, 561-573. doi: 10.2307/3546376

Callaway, R. M., and Tyler, C. (1996). Facilitation in rangelands: direct and indirect effects. In: 'People and rangelands: building the future. Proceedings of the VIth International Rangelands Congress'. Vol. 1. (Eds D. Eldridge and D. Freudenberger.) pp. 197-202. (VI International Rangeland Congress Inc.: Townsville.)

Callaway, R. M., Nadkarni, N. M., and Mahall, B. E. (1991). Facilitation and interference of Quercus douglasii on understory productivity in central California. Ecology 72, 1484-1499. doi: 10.2307/1941122

Choler, P., Michalet, R., and Callaway, R. M. (2001). Facilitation and competition on gradients in alpine communities. Ecology 82, 3295-3308.

Christie, E. K. (1975). A note on the significance of Eucalyptus populnea for Buffel grass production in infertile semi-arid rangelands. Tropical Grasslands 9, 243-246.

Colwell, J. D. (1965). An automatic procedure for the determination of phosphorus in sodium hydrogen carbonate extract of soil. Chemistry \& Industry 10, 893-895.

Dick, R. S. (1975). A map of the climates of Australia. Queensland Geographical Journal 3, 333-369.

Edwards, K. (1979). 'Rainfall in New South Wales: with special reference to soil conservation.' (Soil Conservation Service: Sydney.)

Facelli, J. M., and Pickett, S. T. A. (1991). Plant litter: light interception and effects on an old-field plant community. Ecology 72, 1024-1031. doi: $10.2307 / 1940602$

Fensham, R. J., and Butler, D. W. (2003). Spatial pattern of dry rainforest colonizing unburnt Eucalyptus savanna. Austral Ecology 28, 121-128.

Florentine, S. K. (1999). Ecology of Eucalyptus victrix in grassland in the floodplain of the Fortescue River. PhD thesis, Curtin University of Technology, Perth, Australia.

Funes, G., Basconcelo, S., Diaz, S., and Cabido, M. (2001). Edaphic patchiness influences grassland regeneration from the soil seed-bank in mountain grasslands of central Argentina. Austral Ecology 26, 205-212. doi: $10.1046 / \mathrm{j} .1442-9993.2001 .01102 . x$ 
Hastwell, G. T. (2001). Facilitation and fertile islands: linking canopy effects with plant interactions. $\mathrm{PhD}$ thesis, University of Adelaide, Adelaide, Australia.

Hastwell, G. T., and Facelli, J. M. (2003). Differing effects of shadeinduced facilitation on growth and survival during the establishment of a chenopod shrub. Journal of Ecology 91, 941-950. doi: 10.1046/j.13652745.2003.00832.x

Haworth, K., and McPherson, G. R. (1994). Effects of Quercus emoryi on herbaceous vegetation in a semi-arid savanna. Vegetatio 112, 153-159. doi: 10.1007/BF00044689

Haworth, K., and McPherson, G. R. (1995). Effects of Quercus emoryi trees on precipitation distribution and microclimate in a semi-arid savanna. Journal of Arid Environments 31, 153-170. doi: 10.1006/jare.1995.0057

Heinemann, K., and Kitzberger, T. (2006). Effects of position, understorey vegetation and coarse woody debris on tree regeneration in two environmentally contrasting forests of north-western Patagonia: a manipulative approach. Journal of Biogeography 33, 1357-1367. doi: 10.1111/j.1365-2699.2006.01511.x

Ibáñez, I., and Schupp, E. W. (2001). Positive and negative interactions between environmental conditions affecting Cercocarpus ledifolius seedling survival. Oecologia 129, 543-550.

Jones, R. (1972). Comparative studies of plant growth and distribution in relation to waterlogging. VII. The influence of water-table fluctuations on iron and manganese availability in dune slack soils. Soil Science $\mathbf{6 0}$, 131-139.

Jones, R. (1973). Comparative studies of plant growth and distribution in relation to waterlogging. V. The uptake of iron and manganese by dune and dune slack plants. Soil Science 61, 107-116.

Kennard, D. G., and Walker, B. H. (1973). Relationships between tree canopy cover and Panicum maximum in the vicinity of Fort Victoria. Rhodesia Agricultural Journal 11, 145-153.

Kinloch, J. E., and Freidel, M. H. (2005a). Soil seed reserves in arid grazing lands of central Australia. Part 2. Availability of 'safe sites'. Journal of Arid Environments 60, 163-185. doi: 10.1016/j.jaridenv.2004.03.006

Kinloch, J. E., and Freidel, M. H. (2005b). Soil seed reserves in arid grazing lands of central Australia. Part 1. Seed bank and vegetation dynamics. Journal of Arid Environments 60, 133-161. doi: 10.1016/j.jaridenv.2004.03.005

Ludwig, F., de Kroon, H., Prins, H. H. T., and Brendse, F. (2001). Effects of nutrients and shade on tree-grass interactions in an east African savanna. Journal of Vegetation Science 12, 579-588. doi: 10.2307/3237009

Muñoz, F., and Beer, J. (2001). Fine root dynamics of shaded cacao plantations in Costa Rica. Agroforestry Systems 51, 119-130. doi: 10.1023/A:1010651203815

Oksanen, J., Kindt, R., Legendre, P., and O'Hara, B. (2006). Vegan: community ecology package. R package Version 1.6-9. Available at: http://cran.r-project.org.

Perrolf, K., and Sandström, K. (1995). Correlating landscape characteristics and infiltration - a study of surface sealing and subsoil conditions in semiarid Botswana and Tanzania. Geografiska Annaler, Series A. Physical Geography 77, 119-133.

Pressland, A. J. (1973). Rainfall partitioning by an arid woodland (Acacia aneura F. Muell.) in south-western Queensland. Australian Journal of Botany 21, 235-245. doi: 10.1071/BT9730235

Pressland, A. J. (1976). Soil moisture redistribution as affected by throughfall and stemflow in an arid zone shrub community. Australian Journal of Botany 24, 641-649. doi: 10.1071/BT9760641

Pugnaire, F. I., and Haase, P. (1996). Facilitation between higher plant species in a semi-arid environment. Ecology 77, 1420-1426. doi: $10.2307 / 2265539$

Pugnaire, F. I., and Lázaro, R. (2000). Seed bank and understorey species composition in a semi-arid environment: the effect of shrub age and rainfall. Annals of Botany 86, 807-813. doi: 10.1006/anbo.2000.1240
Rayment, G. E., and Higginson, F. R. (1992). 'Australian laboratory handbook of soil and chemical methods.' (Inkata Press: Melbourne.)

Reinhart, K. O., Maestre, F. T., and Callaway, R. M. (2006). Facilitation and inhibition of seedlings of an invasive tree (Acer platanoides) by different tree species in a mountain ecosystem. Biological Invasions 8, 231-240. doi: 10.1007/s10530-004-5163-9

Rousset, O., and Lepart, J. (1999). Shrub facilitation of Quercus humilus regeneration in succession on calcareous grasslands. Journal of Vegetation Science 10, 493-502. doi: 10.2307/3237184

Rousset, O., and Lepart, J. (2000). Positive and negative interactions at different life stages of a colonizing species (Quercus humilis). Journal of Ecology 88, 401-412. doi: 10.1046/j.1365-2745.2000.00457.x

Royal Botanic Gardens Sydney (2007). PlantNET: the plant information network system of the Botanic Gardens Trust. Version 2.0. Available at: http://plantnet.rbgsyd.nsw.gov.au (accessed 15 June 2007).

Sandquist, D. R., and Ehleringer, J. R. (1998). Intraspecific variation of drought adaptation in brittlebush: leaf pubescence and timing of leaf loss vary with rainfall. Oecologia 113, 162-169. doi: $10.1007 / \mathrm{s} 004420050364$

Searle, P. L. (1984). The benthelot or indophenol reaction and its use in the analytical chemistry of nitrogen. A review. The Analyst 109, 549-568. doi: 10.1039/an9840900549

Shaukat, S. S., and Siddiqui, I. A. (2004). Spatial pattern analysis of seeds of an arable soil seed bank and its relationship with above-ground vegetation in an arid region. Journal of Arid Environments 57, 311-327. doi: 10.1016/S0140-1963(03)00112-5

Silvertown, J., and Wilson, J. B. (1994). Community structure in a desert perennial community. Ecology 75, 409-417. doi: 10.2307/1939544

Simpson, E. H. (1949). Measurement of diversity. Nature 163, 688.

Slatyer, R. O. (1965). Measurements of precipitation interception by an arid zone plant community (Acacia aneura F. Muell.). Arid Zone Research 25, 181-192.

Smit, C., Vandenberghe, C., den Ouden, J., and Müller-Schärer, H. (2007). Nurse plants, tree saplings and grazing pressure: changes in facilitation along a biotic environmental gradient. Oecologia 152, 265-273. doi: 10.1007/s00442-006-0650-6

Song, Y., Kirkham, M. B., Ham, J. M., and Kluitenberg, J. G. (2000). Rootzone hydraulic lift evaluated with the dual-probe heat-pulse technique. Australian Journal of Soil Research 38, 927-935. doi: 10.1071/SR99096

Sthultz, C. M., Gehring, C. A., and Whitham, T. G. (2007). Shifts from competition to facilitation between a foundation tree and a pioneer shrub across spatial and temporal scales in a semi-arid woodland. New Phytologist 173, 135-145. doi: 10.1111/j.1469-8137.2006.01915.x

Taylor, J. A., and Hedges, D. A. (1984). Some characteristics of the trees used by sheep for diurnal camping and differences between the shade and nocturnal camps in a paddock on the northern tablelands of New South Wales. Australian Rangeland Journal 6, 3-9. doi: 10.1071/RJ9840003

Tiedemann, A. R., and Klemmedson, J. O. (1973). Effect of mesquite on physical and chemical properties of the soil. Journal of Range Management 26, 27-29.

Vetaas, O. R. (1992). Micro-site effects of trees and shrubs in dry savannas. Journal of Vegetation Science 3, 337-344. doi: 10.2307/3235758

Walkley, A., and Black, I. A. (1934). An examination of the Degtjareff method for determining soil organic matter, and a proposed modification of the chromic acid titration method. Soil Science 37, 29-38. doi: 10.1097/00010694-193401000-00003

Whitford, W. G. (2002). 'Ecology of desert systems.' (Academic Press: San Diego.)

Zou, C. B., Barnes, P. W., Archer, S., and McMurtry, C. R. (2005). Soil moisture redistribution as a mechanism of facilitation in savanna treeshrub clusters. Oecologia 145, 32-40. doi: 10.1007/s00442-005-0110-8 